\section{A Pascal function for presenting MacPaint files on the Macintosh}

\author{
PETER JORGENSEN, THOMAS J. TUCKER, \\ and JAMES H. REYNOLDS \\ Colgate University, Hamilton, New York
}

Psychological experiments often require the presentation of pictures or other complex graphics as stimuli, and also require control over the time and order of their presentation. If a Macintosh computer is used as a laboratory device, construction of virtually any graphic or pictorial image, as well as text, can be accomplished easily with any of several programs that create paint files (e.g., MacPaint, HyperCard, FullPaint). If one can then write a computer program that will select a given paint file and present it on the computer screen, the basic ingredients for laboratory use and control of graphic stimuli become available. In this paper, we describe a Pascal function useful for presenting any paint image on the screen, and illustrate its possible use.

GETMACPAINT (see Appendix, Lines 15-68) is a boolean function with two arguments: the name of a paint file and the memory location of a bitmap area to which the file contents are to be transferred. It uses the local function GoodFile (Lines 29-37) to test if the file to be opened is a paint file, and the local function FileError (Lines 23-28) to handle errors that may occur when various file-handling procedures are called. If any errors occur, GETMACPAINT is exited with a value of "false." GETMACPAINT's statement part uses a set of nested "if" statements that perform the following tasks unless a file-handling or a memory-allocation error occurs.

1. Use GoodFile to verify that the file name (parameter fNAME) to be opened is a paint file (Line 40);

2. Open the paint file fName using FSOPEN (Line 41);

3. Find the end of the file (using GETEOF, Line 43) and establish a starting position (MacPaint's first allocation block, 512 bytes, does not contain any part of the picture);

4. Use SETFPOS to locate the point in the file at which reading begins and allocate a data structure to hold the data and a pointer to it (Lines 46-49);

5 . Read the file row by row into the bitmap using UNPACKBITS (Lines 50-60; MacPaint bytes are packed but bitmap bytes are not, so unpacking is necessary);

6. Set the value of the function to "true" and dispose of the file-reading pointer using DISPOSPTR (Lines 61-62);

7. Close the file using FSCLOSE (Line 66).

Correspondence may be addressed to James H. Reynolds, Psychology Department, Colgate University, Hamilton, NY 13346.
The Appendix lists a short program that illustrates the use of GETMACPAINT. This skeleton backwardmasking program is designed simply to present a singlegraphic test stimulus on the Macintosh screen for a short period $($ minimum of 1 clock tick $=16.625 \mathrm{msec}$; see $I n$ side Macintosh, 1985, Vol. 3, p. 19) then replace it with a masking stimulus in the same screen position. Both the test stimulus and the masking stimulus are assumed to have been stored in paint files named PICl and PIC2, respectively, which must be in the same folder as the program. The program will run in Turbo Pascal or Lightspeed Pascal (see modification comments in the listing). Several of the graphics and bitmap procedures used are modifications of those described in Swan (1987, pp. 100-109), and their details are well documented there. Here we give a brief and nontechnical summary to help make the program more comprehensible.

The main program (Lines 137-157) performs preliminary initializations and then calls the procedure DOGRAPHICS, which runs the experiment. The LM and RM (Lines 142-151) variables adjust the size of the image, depending on whether a Macintosh SE or Macintosh II screen is being used. The main program also sets the display time for the stimulus ( $P$, in Line 152) and defines the width and number of the image bands that DOGRAPHICS will display on the screen.

DOGRAPHICS (Lines 94-136) calls the procedure InitBitMap (Lines 69-79) twice, which uses QuickDraw's bitmap data structure (a record-type) to create two bitmap areas that will contain paint images for a test stimulus (Line 102) and a masking stimulus (Line 103). It then calls the procedure SetUpPic (Lines 80-93) twice, using as parameters the name of the GETMACPAINT files to be read (PIC1 and PIC2, respectively, in the example) and the bitmap area in which each file's image is to be stored.

The SetUpPic procedure performs several bitmap operations. First, it calls InitBitMap (Line 83) to construct a bitmap that will hold the entire image that the GETMACPAINT function reads from a file. GETMACPAINT then reads the file into this bitmap (Line 86). Next, this image is moved (using BlockMove, Line 89) to the predefined bitmap area identified by the parameter BM in the SetUpPic's argument list. This strategy allows the programmer to read a paint file and store it either temporarily or permanently in a well-defined area that may vary in size from the original image. In the present example, the bitmaps PIClbits and PIC2bits were defined to be the same size as the file image being read (see Lines 102 and 103), but they could have been reduced in size by giving smaller parameters that would hold only part of the original image.

After using SetUpPic to read paint images into the PIClbits and PIC2bits bitmaps, in Lines 106-116 DOGRAPHICS opens a GrafPort for drawing an image, 
blanks it out, and waits for the beginning of the next screen refresh. It then uses QuickDraw's SetRect and CopyBits procedures (Lines 117-122) to display the contents of the PIClbits bitmap on the screen as a series of image bands that are copied from the top to the bottom of the screen in succession ${ }^{1}$ and waits for a specific number of ticks (Lines 123-124, and see Line 152, in which the variable was defined) before replacing the image with the mask. Finally, the code in Lines $125-136$ presents the mask image stored in the PIC2bits bitmap, waits until the mouse button is pressed, then closes the port and ends the program.

\section{Additional Presentation Details}

Precise location of all or part of a paint file presented on the screen can be achieved in several ways. If, for example, the entire window of a MacPaint file is to be presented on a Macintosh SE screen, the parameters LM and RM shown in Lines 149-150, and values of 60, 0 , and 5 inserted in Lines 153-155, respectively, can be used. To change the positioning of this entire image on the screen, the second parameter in the SetRect procedure in Line 77 will move the image to the left (minus) or right (plus), and the third parameter will move it up (minus) or down (plus). If only a part of the total image in a MacPaint screen is to be displayed, Lines 149-150 can be adjusted to cut down the left and right sides of the total image, and Lines 153-155 will narrow the vertical aspect of the field. With a little practice at manipulating these parameters, and with appropriate positioning of the image when it is placed in the MacPaint window, the user can direct the program's placement of an image to virtually any place desired on the output screen.

\section{Availability}

This program is available on a 3.5-in. floppy disk. The software is free, but a charge of $\$ 4.00$ is requested for postage and handling. Send a formatted diskette and a check to J. H. Reynolds, Psychology Department, Colgate University, Hamilton, NY 13346.

\section{REFERENCES}

Inside Macintosh. (1985). Vols. 1-3. Reading, MA: Addison-Wesley. Swan, T. (1987). Programming with Macintosh Turbo Pascal. New York: Wiley.

\section{NOTE}

1. These bands (whose vertical height and number are defined in Lines 153-155) are used to divide the bitmap into strips for rapid copying. The copybits procedure is limited to copying bitmaps that are at most half the size of the stack (about $3 \mathrm{~K}$ ). An entire screen is about $140 \mathrm{~K}$, so it must be copied in sections to avoid stack overflow.

\section{APPENDIX}

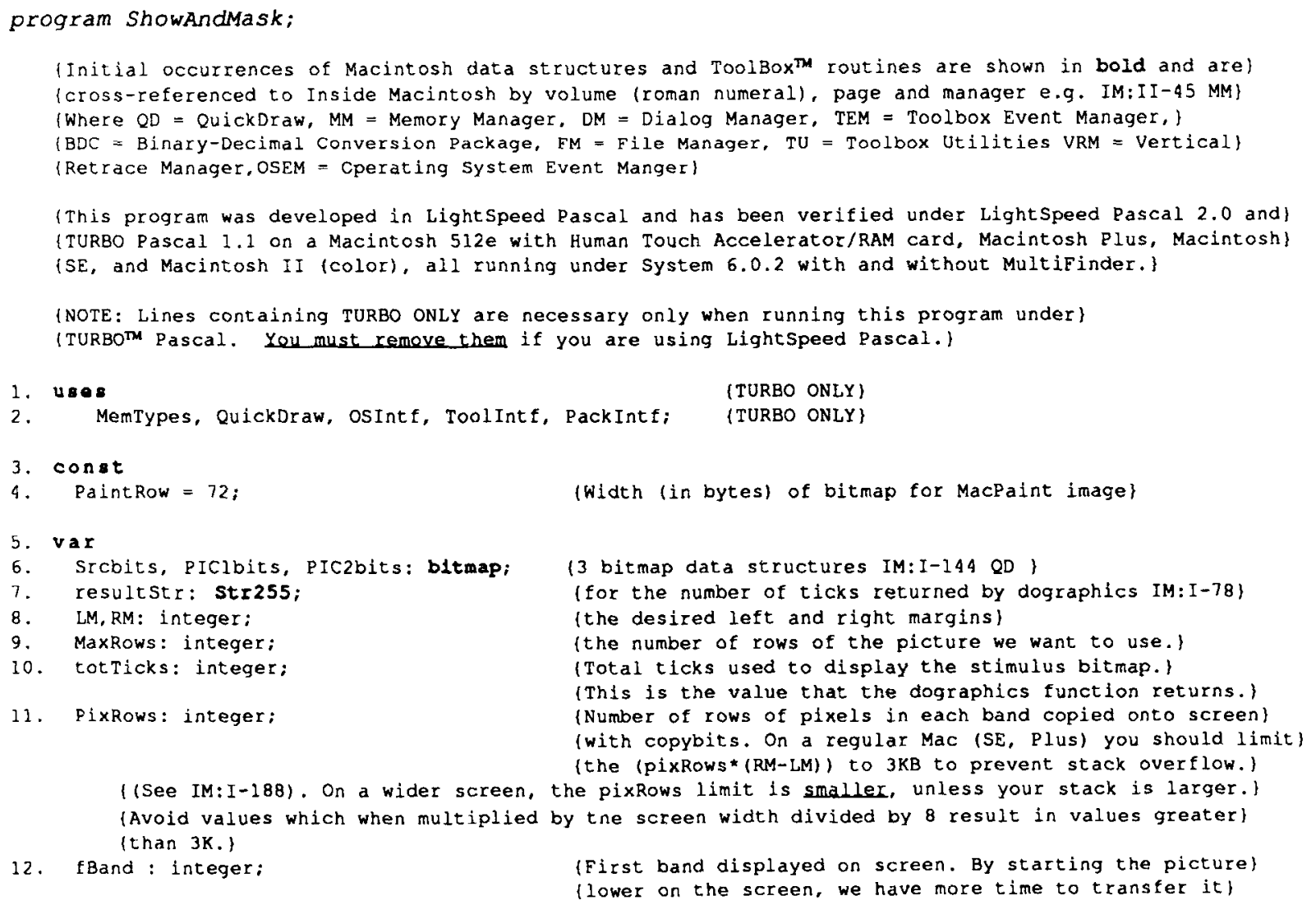


13. lastBand: integer;

14. P : integer; (before the scan line catches up with our copybitting.)

(Number of bands of pixRows rows to be displayed on screen.) (We must transfer the image to the screen in sections not exceeding $3 \mathrm{~KB}$ ) (in size, so we transfer in bands of pixRows height and RM-LM width.) (We can oniy get so much on the screen in one refresh. Different machines) \{have different capabilities depending on *refresh rate, *processor speed, ) \{'memory, etc.\}

(The desired pause (in ticks) during which PICl remains on screen.)

15. function getmacpaint (fName: str255; var bm: bitmap): boolean;

(Design and coding by Peter Jörgensen, Colgate University Computer Center.\}

TOpens a MacPaint file and unpacks it into the bitmap SRCBITS.)

\{This function contains several nested functions and procedures so that it can be incorporated into\} \{a program in it's entirety without losing any of it's functionality. It returns TRuE if successful\} (and FALSE if unsuccessful - for any reason.?

16. conat

17. MPaintTYPE = 'PNTG':

\{Files must be painting files.\}

18.

va r

packedbitsptr, srcptr, destptr: ptr;

startpos, fileLen: longint;

refnum, row: integer;

theError:integer;

\{File length and where to start.\}

(ID number for file and a looping int)

(For the result code returned by FSCLOSE at the end.)

23. function FileError (theErr: integer, name: str255): boolean;

\{Returns false if a file error is encountered.)

\{This function could be expanded to show an Alert informing the user of the nature of the\} (error and the name of the file (name). This is especially useful during the debugging) \{process.\}

24. $\operatorname{bog} 1 \mathrm{n}$

25. FileError := true;

26. if theErr $=0$ then

27 .

28 .

FileError := false;

end;

29. function GoodFile (fileName: str255): boolean;

(This function checks to see if a file is of the correct type ('PNTG'). It returns "FALSE" )

(if it isn't. There are several programs which can create PNTG files, we want to be able to use b

lany of them.\}

30.

var

fndrinfo: f1nfo: \{IM: II-84 FM\}

$\operatorname{bog} 1 \mathrm{n}$

Goodfile := false;

if not (FileError(Gotrinfo(fileName, 0, fndrinfo), fileName)) then (IM:II-95 FM)

if (fndrInfo.fdtype = MPaintTYPE) then

GoodFile : = true:

end;

beg1n

getmacpaint := false;

if Goodfile (fName) then

if not FileError (FSOPEN (fName, O, RefNum), fName) then (IM:II-91 FM)

(We successfully opened the file which will be referred to by RefNum from now on.\} begin

if not FileError (GETzor(RefNum, fileLen), fName) then \{IM:II-93 FM\} begin

(Store the length of the file in filelen)

44.

45.

46.

47.

48.

49.

50 .

51.

52.

53.

54.

55.

56.

57.

startpos := 512 ;

if not FileError (SETrPOS(RefNum, farrometart, startpos), fName) then (IM: II-93 FM) begin

(Skip the first allocation block (512 bytes) - it's not part of the picture)

filelen := filelen - startpos; (Calc the length of the pertinent data.)

packedbitsptr := newptr(fileLen); (Allocate a block of memory of this size.IM:II-36 MM]

if packedbitsptr $\longleftrightarrow$ nil then

(Memory allocation was successful.) begin

if not FileError (FSREAD (RefNum, fileLen, packedbitsptr), fName) then (Read the file into memory IM:II-92 FM)

begin

srcptr: = packedbitsptr;

destptr $:=$ bm, baseaddr;

for row $:=1$ to MaxRows do

begin

(Fill scrbits bitmap with unpacked bits from PTNG file.) 
58.

59.

60.

61.

62.

63.

64.

65.

66.

67.

68.

69. and ;
ONPACKBITS (srcptr, destptr, PaintRow) : (IM:II-470 TU)

(The bytes in which a picture is stored in a MacPaint file are packed, ) (displayed bitmaps are not, so we unpack them into a new datastucture.)$$
\text { end; }
$$$$
\text { end; }
$$

\{unpacking the bits\}

(reading in data)

\author{
(IM:II-36 MM) \\ (allocating packedbitsptr) \\ (setting FPos and all that stuff) \\ \{finding EOF) \\ (Close the file. IM:II-94 File Manager) \\ (file opened) \\ lof function)
}

procedure initBitmap (var bm: bitmap; width, height: integer);

(This procedure initalizes a bit map in which each row contains width bytes and there are height) \{rows. Width should be divisible by 2 to optimize the copybits process (the data should be aligned) (on word boundaries\}.)

70. $\operatorname{bog} 1 \mathrm{n}$

71. if width mod $2=1$ then

72 .

73.

74 .

75.

76.

(Width is the number of pixels/8 which we want to display.) baseaddr := newptr(longint (width) * longint (height)): (IM:II-36 MM)

isince the product of width and height may be greater than the largest integer, put width) \{and height into 4 byte longints.\}

eetrect (bounds, 0,0 , (width $*$ ), height); (setrect takes parameters in bits, so multiply) (width (bytes) by 8 IM:I-174 QD) \{The second and third parameters to setrect can\} (be varied to reposition the original Macpaint) (image on the presentation screen.)

78.

79.

80.

81.

82.

83.

84.

85

86.

87 .

88 .

89.

90.

91.

92 .

93.

94.

end

and:

function SetUpPic (var bm: bitmap; fileName: string): boolean; (Loads a picture into bm)

bog $1 \mathrm{n}$

SetUpPic: = false

initbitmap (srcbits, paintRow, screenbits.bounds.bottom): (Allocate a bitmap data structure to hold) \{the plcture.\}

if srcbits.baseaddr $\ll$ nil then

(Allocation was successful.

begin

if GetMacPaint (fileName, srcbits) then

(Success in reading and unpacking bitmap.)

begin

Setuppic := true;

BlockMove (srcbits.baseaddr, bm.baseaddr, GetPtrsize(srcbits.baseaddr)); 1Copy the unpacked bitmap into bm. IM:II-44 MM \& IM:II-37 MM

\{Don't use CopyBits here as the data structures are too large. IM:I-188 QD

end:

disposptr (srcbits.baseaddr);

\{Free the block allocated for srcbits\}

end;

function dographics (SFile, MFile: str255; bheight, fBand, lastBand, LM, RM, pause: integer): integer; (Shows SFile, then MFile returns the number of ticks that it took to display SFile.)

(The size and location of the images are determined by the following parameters:\}

(Top of image fBand ${ }^{2}$ ixRows

(Bottom of image (lastBand + EBand) $* P i \times R o w s$ )

(Left side LM)

\{Right side RM\}

95.

96.

98

98.

100 .

var

startTick, EndTick, finalTick: longint:

tTickstr: str255;

myGPtr: GrafPtr;

band: integer;

bandRect: rect:

101. $b \circ g 1 n$

102. initbitmap (PIClbits, 72, Maxrows):

103. initbitmap (PIC2bits, 72, Maxrows)

104. if SetUpPic (PIClbits, SFile) then

[Define bitmap for stimulus\}

[Define bitmap for mask\}

(Put PICl file into PIClbits) 
APPENDIX (continued)

122.

123.

124.

125.

126.

127.

128 .

129.

130.

131.

132.

133.

134.

135.

136. end; if SetUpPic(PIC2bits, MFIle) then begin

MyGPtr := GrafPtr(NewPtr(sizeof (GrafPort)));
(Put PIC2 file into PIC2bits)

(Allocate space in the heap for our grafPort.) (IM: I-163. QD)

(Create a grafport to do our drawing in. IM:I-163 OD) (Make the port white. IM:I-177 QD)

(Set up a 1 second delay on white screen. IM:I-260 TEM)

(Do nothing until the time has passed.
Openport (MyGPtr);

f111rect (MyGPtr ${ }^{n}$ portrect, white);

startTick : = T1ckCount +60 ;

repeat

until starttick < TickCount;

starttick := TickCount;

repeat

until starttick $\gg$ TickCount;

starttick := TickCount;

for band $:=$ fBand to lastBand do

begin (Put PICl picture on screen in sections that won't overflow the stack.) setRect (bandRect, LM, band * bheight, RM, band * bheight + bheight); (IM:I-174 QD) copybit»(PIClbits, MyGPtr^portBits, bandRect, bandRect, srccopy, nil); (IM:I-188 QD) end;

Ooptimization note: By reducing the amount of math performed during the bit copying\{e.g. by predefining the series of band related parameters (band * bandheight), \} (looping through the setRect procedure to create several rectangles before using \} \{several copybits statements instead of a loop - a larger picture can be presented in\} \{one screen refresh. The use of constants for these parameters will also speed up ) the process at the cost of increasing the coding and decreasing the flexibility of , the function. Compiling with Debugging and Range checking off also speeds it up.)

endTick := TickCount;

repeat

until endTick + pause < tickCount; (Wait the requested number of ticks before continuing.)

for band := fBand to lastBand do

begin. (Put PIC2 picture on screen. \}

setRect (bandRect, LM, Land * bheight, RM, band * bheight + bheight);

copybits (PIC2bits, MyGPtr^portBits, bandRect, bandRect, srccopy, nil): end;

dographics := endTick - StartTick;

(Dographics returns the time (in ticks) that it took to)

ipresent PICl. If this time is 0 , then we know that the

\{presentation took place entirely within one screen) \{refresh.\}

repeat

\{Wait until the mouse button is pressed. Remove lines 131\}

\{to 132 if control is to be returned to caller immediately)

\{IM: I-259 TEM\}

\{Close our grafport IM:I-164 QD \}

until Button;
Closeport (MYGPtr):

\{and dispose of its storage. IM:II-36 MM)

Disposptr(Ptr(MyGPtr)); end;
137. beg1n (nAIN PROGRAM -

138. In1tgraf (etheport);

139. hidecureor:

140. FlushEvents (everyevent, 0$)$;

141. MaxRows := acroenb1ta.bounds.bottom;

142. if screenbits.bounds.right $>576$ then

143.

144 .

145 .

146.

147.

148 .

149.

150.

151 .

begin

LM $:=$ (screenbits.bounds.right -576$)$ div 2 ;

$R M:=L M+576$

end

else

begin

LM : $=0$;

$\mathrm{RM}:=$ screenbits.bounds.right;

for just display the left hand side of the Macpaint picture;

\{TURBO ONLY IM:I-162\}

$\{$ No distractions IM:I-168 OD

(Get rid of old clicks, etc. IM:II-69 OSEM)

\{Only unpack as much picture as we can fit on screen.\}

$\{I M: I-143$ QD $\}$

\{Center 8 inch wide MacPaint picture on larger screens; end;
152. $P:=60$;

153. PixRows: $=40$;

154. fBand $:=2$;

155. lastBand $:=5$;

156. totTicks := (dographics('PICl',
$\{$ or...\}

\{LM and RM can be varied to display a narrower image, if desired.\} (but remember to keep the margins on word (even byte) ) (boundaries for fastest performance.\}

(Set the desired pause in ticks.)

(Set this var to the desired height of the bands copied.\}

(Set this var to the desired first band of the image.\}

(Set this var to the number of bands of image to be displayed.) end.
PIC2', pixRows, fBand, lastBand, LM, RM, P)); (Show the pictures) 